\title{
Classical BRST Cohomology and Invariant Functions on Constraint Manifolds. I
}

\author{
Michael Forger and Johannes Kellendonk $\star$ \\ Fakultät für Physik der Universität Freiburg, Hermann-Herder-Straße 3, W-7800 Fŕeiburg, \\ Federal Republic of Germany
}

Received July 25, 1990

\begin{abstract}
Given a manifold $M$ and a submanifold $C$, together with a Lie group $G$ acting on $M$ and leaving $C$ invariant, it is shown how the algebra of $G$-invariant functions on $C$ can be described in terms of cohomology when $C$ is defined as the common zero level of an irreducible set of ( $G$-covariant) constraints. The construction is independent of any additional structures such as, e.g., a symplectic structure on $M$, and therefore it provides a natural framework for a unified description of BRST cohomology both for Lagrangian and Hamiltonian systems. Finally, it is discussed how one can, in various typical situations, replace invariance under an infinite-dimensional gauge group by invariance under a suitable finitedimensional Lie group; this is a necessary prerequisite for handling BRST cohomology for such systems within a completely finite-dimensional setting.
\end{abstract}

\section{Introduction}

Among the various approaches towards the problem of quantizing classical dynamical systems, both in mechanics and in field theory, the method now commonly called BRST quantization is the most recent one. In contrast to other, more traditional techniques such as, e.g., canonical quantization or geometric quantization, the BRST approach is specifically designed to deal with singular dynamical systems, or to be more precise, with dynamical systems whose singular nature is due to the presence of a local (gauge) symmetry (these are the only ones that are of relevance to physics and will in the sequel be simply referred to as gauge theories). The basic idea is to introduce additional unphysical degrees of freedom (ghosts) and to replace the original local (gauge) symmetry by a global (super)symmetry (the BRST symmetry), generated by a single operator (the BRST operator) whose square vanishes and which therefore defines a cohomology theory

\footnotetext{
* New address: Physikalisches Institut der Universität Bonn, Nußallee 12, W-5300 Bonn, FRG
} 
(the BRST cohomology). This BRST operator appeared for the first time in the pioneering work of Becchi et al. [4] on the quantization of Yang-Mills theories by means of functional integrals à la Faddeev-Popov [8]. However, it soon became clear that the concept is really much more general and that such an operator should exist in any gauge theory - irrespective of whether one is dealing with infinitely many degrees of freedom (as in field theory) or with finitely many degrees of freedom (as in mechanics) and also whether one is looking at the classical theory or at the quantum theory. In all cases, the main role of the BRST operator is to single out the observables and the physical states within the theory.

In the present paper, we shall concentrate on classical BRST cohomology for gauge theories with a finite number of degrees of freedom. Even in this restricted context, there already exist different versions of the same basic idea, and so it seems natural to ask for a unified mathematical framework which can serve as a common denominator for all of them. One approach to the subject, which has been the starting point for our investigation, is that of Kostant and Sternberg [13]: they give the mathematical foundation for the BRST procedure in the context of classical finite-dimensional Hamiltonian systems with symmetry (Hamiltonian $G$-spaces) and relate it to symplectic reduction. Our work has grown out from an attempt to a) understand the precise regularity conditions under which the BRST cohomology, as introduced in [13], does yield the algebra of functions on the reduced phase space and b) investigate how the construction in [13] can be extended to encompass not only (constrained) Hamiltonian systems but also (degenerate) Lagrangian systems: this necessarily involves generalizing the BRST procedure in such a way that it no longer depends on the symplectic structure of phase space.

On the other hand, Henneaux et al. have recently published a series of papers $[10,11,12]$ on classical BRST cohomology for constrained Hamiltonian systems which do generalize the construction in [13], though in a somewhat different direction. Common to their approach and to ours is that one is always dealing with a manifold $M$ and a submanifold $C$ defined by some set of constraints; these can be assembled into a single vector-valued function $\phi: M \rightarrow V^{*}$ such that $C$ appears as the zero level of $\phi$ :

$$
C=\phi^{-1}(0) \text {. }
$$

(Normally, one introduces a basis $\left\{v_{i}\right\}_{i}$ of $V$ and represents $\phi$ by its components $\phi_{i}$ with respect to the dual basis $\left\{v^{i}\right\}_{i}$ of $V^{*}$.) In addition, one has to impose some regularity condition, namely that $0 \in V^{*}$ is (at least) a weakly regular value of $\phi$.

To explain this in more detail, recall first that $0 \in V^{*}$ is called a regular value of $\phi$ if for every point $m \in C$, the tangent map $T_{m} \phi$ to $\phi$ at $m$, as a linear map from the tangent space $T_{m} M$ of $M$ at $m$ to the vector space $V^{*}$, is onto. As is well known, this condition forces $C$ to be a submanifold of $M$ and implies that for every point $m \in C$, the tangent space $T_{m} C$ of $C$ at $m$ coincides with the kernel of $T_{m} \phi$ in $T_{m} M$ :

$$
T_{m} C=\operatorname{ker} T_{m} \phi .
$$

But often the condition of regularity is too strong, and one would like to weaken it. The simplest way to do this is to just use the consequences of regularity 
mentioned before as part of a new definition. Correspondingly, $0 \in V^{*}$ is called a weakly regular value of $\phi$ if

- $C$ is a submanifold of $M$,

- for every point $m \in C$, the tangent space $T_{m} C$ of $C$ at $m$ coincides with the kernel of $T_{m} \phi$.

(Note that if one just requires $C$ to be a submanifold of $M$, then for every point $m \in C$, the tangent space $T_{m} C$ of $C$ at $m$ is automatically a subspace of the kernel of $T_{m} \phi$ in $T_{m} M$ :

$$
T_{m} C \subset \operatorname{ker} T_{m} \phi .
$$

Thus it is only the opposite inclusion which has to be required as an additional condition.)

In more familiar terms, we shall call the set of constraints formed by the $\phi_{i}$ irreducible respectively reducible when $0 \in V^{*}$ is a regular value respectively a weakly regular but not regular value of $\phi$.

The main difference between the approach of Henneaux et al. and the one taken here is now, roughly speaking, that these authors deemphasize the group-theoretical aspects but make strong use of symplectic geometry, while we do exactly the opposite: our constructions rely heavily on group-theoretical methods but do not make any reference to symplectic geometry. This means that in addition to the hypotheses made above, Henneaux et al. assume $M$ to be a symplectic manifold and $C$ to be a coisotropic submanifold (i.e., the constraints are first class), while we suppose $M$ to carry an action and $V^{*}$ to carry a representation of a Lie group $G$ such that the map $\phi$ becomes $G$-covariant. In our view, each of the two approaches has its own advantages and its own drawbacks; for example, the symplectic method extends naturally to the so-called open gauge algebras (characterized by the fact that the Poisson brackets between the constraints involve structure functions rather than structure constants), while the group-theoretical technique is directly applicable to the Lagrangian framework as well. Of course, the two approaches have a large overlap because they can both be applied to Hamiltonian $G$-spaces with $\phi$ as the momentum map [1]: this is the scenario envisaged by Kostant and Sternberg [13]. A complete comparison is however only possible in the regular (irreducible) case, that is, when the action of $G$ on $C$ is required to be almost free (cf. Sect. 4), because only then does the construction in [13] produce the correct BRST operator.

The plan of the paper is as follows. In Sect. 2, we explain the construction of the BRST double complex and use a contracting homotopy operator to prove that the cohomology of the BRST operator in degree 0 reproduces the $G$-invariant functions on the constraint manifold $C$. In doing so, we explicitly assume that we are dealing with an irreducible set of constraints (regularity). Generalization to a reducible set of constraints (weak regularity) is possible, but the details are quite complicated and will hopefully be presented in a future publication. In Sects. 3 and 4, we discuss applications of the general construction to Lagrangian systems and Hamiltonian systems, respectively, and in Sect. 5, we show how in some typical cases, gauge invariance can be coded into invariance under an appropriate finite-dimensional Lie group. 


\section{Construction of the BRST Complex}

The present section will be devoted to explaining how the algebra of invariant functions on a constraint manifold can be described in terms of cohomology. Briefly, we shall show that this algebra can be identified with the cohomology in degree zero for the total differential operator $D=d_{1}+d_{2}$ in a double complex $\left(S, d_{1}, d_{2}\right)$, which is obtained as the tensor product of a Lie algebra complex $\left(\Lambda \mathrm{g}^{*}, d_{2}\right)$ with a certain Koszul complex $\left(K, d_{1}\right)$. The whole construction has a purely algebraic aspect as well as a more geometric one; we begin with the former.

First of all, let $\mathfrak{g}$ be a finite-dimensional Lie algebra, let $\Lambda \mathfrak{g}^{*}$ be the Grassmann algebra over its dual $\mathrm{g}^{*}$, and let $d_{2}$ be the unique derivation of degree 1 on $\Lambda \mathrm{g}^{*}$ that satisfies

$$
d_{2} \alpha(\xi, \eta)=-\alpha([\xi, \eta])
$$

for all $\alpha \in \mathfrak{g}^{*}, \xi, \eta \in \mathfrak{g}$. Then $d_{2}^{2}=0$, so $\left(\Lambda \mathfrak{g}^{*}, d_{2}\right)$ is a complex

$$
0 \longrightarrow \mathbb{R} \stackrel{d_{2}}{\longrightarrow} \mathfrak{g}^{*} \stackrel{d_{2}}{\longrightarrow} \cdots \stackrel{d_{2}}{\longrightarrow} \Lambda^{i} \mathfrak{g}^{*} \stackrel{d_{2}}{\longrightarrow} \Lambda^{i+1} \mathfrak{g}^{*} \stackrel{d_{2}}{\longrightarrow} \cdots,
$$

whose cohomology is, by definition, the cohomology of $\mathfrak{g}$ (as a Lie algebra). Incidentally, this Lie algebra complex $\left(\Lambda \mathrm{g}^{*}, d_{2}\right)$ consists of a simply graded (Z्Z-graded), graded commutative algebra, which is freely generated by finitely many generators of degree 1 (and is connected in degree zero, i.e. satisfies $\Lambda^{0} \mathfrak{g}^{*}=\mathbb{R}$ ), together with a differential operator of degree 1, and it is not difficult to show that conversely, every such complex arises from a suitable finite-dimensional Lie algebra (given such a complex, one uses Eq. (4) to define the Lie bracket $[\because, \cdot]$ and deduces the Jacobi identity from $d_{2}^{2}=0$ ).

Next, assume that $\left(K, d_{1}\right)$ is some other complex consisting of a simply graded ( $\mathbb{Z}$-graded), graded commutative algebra $K$, together with a differential operator $d_{1}$ of degree 1 , such that $K$ carries a representation $\rho_{K}$ of $g$ (by derivations of degree 0 ) under which $d_{1}$ is covariant, i.e.

$$
\rho_{K}(\xi)\left(d_{1}(k)\right)=d_{1}\left(\rho_{K}(\xi) k\right)
$$

for all $k \in K, \xi \in \mathfrak{g}$, the most important situation being that where this complex is acyclic, i.e., has trivial cohomology except possibly in degree zero:

$$
H_{d_{1}}^{i}(K)=0 \text { for } i \neq 0 .
$$

Then taking the graded tensor product of $K$ with $\Lambda \mathrm{g}^{*}$, we obtain a graded commutative algebra $S=K \otimes \Lambda \mathrm{g}^{*}$ : in fact, $S$ is doubly graded $((\mathbb{Z} \times \mathbb{Z})$-graded) and hence also simply graded ( $\mathbb{Z}$-graded), namely with respect to the total degree defined as the sum of the two components of the bidegree (graded commutativity refers to this total degree). Explicitly,

$$
S=\bigoplus_{k \in Z} S^{k}, \quad S^{k}=\bigoplus_{i+j=k} S^{i, j}, \quad S^{i, j}=K^{i} \otimes \Lambda^{j} \mathfrak{g}^{*} .
$$

Moreover $d_{1}$ and $d_{2}$ can both be extended to unique differential operators $d_{1}$ of bidegree $(1,0)$ and $d_{2}$ of bidegree $(0,1)$ on $S$ by letting $d_{1}$ act trivially on the second factor (i.e., $d_{1}(k \otimes \alpha)=d_{1}(k) \otimes \alpha$ for all $\left.k \in K, \alpha \in \Lambda g^{*}\right)$ and requiring $d_{2}$ to satisfy

$$
(-1)^{|k|}\left(d_{2} k\right)(\xi)=\rho_{K}(\xi) k
$$


for all $k \in K$ of degree $|k|$ (we write $k$ for $k \otimes 1$ ). In addition, it turns out that $d_{1}$ being $g$-covariant is equivalent to requiring that $d_{1}$ and $d_{2}$ anticommute on $S$ or that $D=d_{1}+d_{2}$ satisfies $D^{2}=0$ on $S$, so under the assumptions made, $\left(S, d_{1}, d_{2}\right)$ is a double complex and $(S, D)$ is a complex. Each of the three differential operators on $S, d_{1}, d_{2}$ and $D$, then defines its own cohomology, $H_{d_{1}}(S), H_{d_{2}}(S)$ and $H_{D}(S)$, which are of course not independent. But although the relation between $d_{1}, d_{2}$ and $D$ is simple, that between the corresponding cohomologies is not: it can be determined by means of a standard but rather complicated technique from algebraic topology known under the name "spectral sequences." In this context, the importance of the requirement that the complex $\left(K, d_{1}\right)$ be acyclic becomes apparent: it lies in the fact that in this case - which is the only one we shall consider from now on - the spectral sequence collapses at the $E_{2}$-term [5], which means that

$$
H_{D}^{k}(S)=H_{d_{2}}^{k}\left(H_{d_{1}}^{0}(S)\right) \text {. }
$$

Here, the equality sign is understood to hold in an abstract sense, that is, up to a canonical isomorphism. Concretely, finding the element in $H_{D}^{k}(S)$ that corresponds to a given element of $H_{d_{2}}^{k}\left(H_{d_{1}}^{0}(S)\right)$ under this isomorphism amounts to explicitly solving the so-called descent equations. In the following, we shall assume that an appropriate choice is made for the complex $\left(K, d_{1}\right)$ (see the discussion below) and call $\left(S, d_{1}, d_{2}\right)$ the BRST double complex, $(S, D)$ the BRST complex and $D$ the BRST operator.

Before going on, we would like to remark that even when we disregard all algebraic structures on $K$, namely the multiplication, the grading and the differential operator $d_{1}$, we still obtain a complex

$$
0 \longrightarrow K \stackrel{d_{2}}{\longrightarrow} K \otimes \mathrm{g}^{*} \stackrel{d_{2}}{\longrightarrow} \cdots \stackrel{d_{2}}{\longrightarrow} K \otimes \Lambda^{i} \mathrm{~g}^{*} \stackrel{d_{2}}{\longrightarrow} K \otimes \Lambda^{i+1} \mathfrak{g}^{*} \stackrel{d_{2}}{\longrightarrow} \cdots
$$

whose cohomology is, by definition, the cohomology of $\mathfrak{g}$ (as a Lie algebra) with coefficients in $K$; in particular, the cohomology in degree 0 gives precisely the $\mathrm{g}$-invariant elements of $K$ :

$$
H_{d_{2}}^{0}(S)=K^{\mathrm{g}} .
$$

In particular, this can be applied with $K$ replaced by $H_{d_{1}}^{0}(K)$, so that we get

$$
H_{d_{2}}^{0}\left(H_{d_{1}}^{0}(S)\right)=H_{d_{2}}^{0}\left(H_{d_{1}}^{0}(K) \otimes \Lambda g^{*}\right)=\left(H_{d_{1}}^{0}(K)\right)^{\mathrm{g}},
$$

where the first equality holds because $d_{1}$ acts trivially on $\Lambda \mathrm{g}^{*}$.

Except for the condition of carrying a representation of the Lie algebra $\mathfrak{g}$, the complex $\left(K, d_{1}\right)$ has so far been left completely arbitrary. Usually, however, it is constructed from more elementary data, suggested by the situation at hand. Here, we shall choose it to be a Koszul complex, given by the following construction: Let $V$ be a finite-dimensional vector space and $R$ be a commutative algebra (with unit), both of which carry representations $\rho_{V}$ and $\rho_{R}$ of $\mathfrak{g}$, respectively, and let $d_{1}: V \rightarrow R$ be a $g$-covariant linear map from $V$ into $R$. Then define $K$ to be the simply graded ( $\mathbb{Z}$-graded), graded commutative algebra $K=R \otimes \Lambda V$ and extend $d_{1}$ to a unique derivation $d_{1}$ of degree 1 on $K$ by letting it act trivially on $R$; thus in particular

$$
d_{1}(r \otimes v)=r d_{1}(v)
$$


for all $r \in R, v \in V$. Then $d_{1}^{2}=0$, so $\left(K, d_{1}\right)$ is a complex

$$
\cdots \stackrel{d_{1}}{\longrightarrow} R \otimes \Lambda^{i} V \stackrel{d_{1}}{\longrightarrow} R \otimes \Lambda^{i-1} V \stackrel{d_{1}}{\longrightarrow} \cdots \stackrel{d_{1}}{\longrightarrow} R \otimes V \stackrel{d_{1}}{\longrightarrow} R \longrightarrow 0
$$

whose cohomology in degree zero is given by

$$
H_{d_{1}}^{0}(K)=R / R d_{1}(V) .
$$

Note that in order to let $d_{1}$ really have degree +1 (and not -1 ), we must consider $K$ to be negatively graded, i.e.,

$$
K^{i}=R \otimes \Lambda^{-i} V
$$

In addition, it should be observed that the given representations $\rho_{V}$ of $g$ on $V$ and $\rho_{R}$ of $\mathfrak{g}$ on $R$ (by derivations) give rise to a representation $\rho_{K}$ of $\mathfrak{g}$ on $K$ (by derivations of degree 0 ) and that $d_{1}$ being covariant as a linear map from $V$ to $R$ forces $d_{1}$ to be covariant as a differential operator on $K$. Thus all that remains to be seen is whether the resulting complex is actually acyclic. The importance of this condition has been explained before, and we will later discuss conditions to be imposed on the map $d_{1}$ in order for that to be the case. But even when $d_{1}$ does have non-trivial cohomology in degree $\neq 0$, there is a way to construct an acyclic complex which is an extension of the complex $\left(R \otimes \Lambda V, d_{1}\right)$ considered here: this method, due to Tate [16], can be applied (at least) as long as $R$ is a Noetherian ring.

Turning to the more geometric part, we now want to show how BRST cohomology can be used to describe the algebra of invariant functions over a constraint manifold, thus bringing algebraic concepts into contact with differential geometric ones. To this end, let $G$ be a connected (real) Lie group with Lie algebra $\mathrm{g}$ and let $M$ be a (smooth real) manifold carrying a (smooth) action of $G$. Let $R$ be the algebra of (smooth real) functions on $M: R=\mathscr{F}(M)$. The action of $G$ on $M$ induces a representation of $\mathfrak{g}$ on $\mathscr{F}(M)$ (by derivations) which is given by associating with each generator $\xi \in \mathfrak{g}$ of $G$ minus the Lie derivative along the corresponding fundamental vector field $\xi_{M}$ on $M$. (The fundamental vector field $\xi_{M}$ corresponding to $\xi$ is defined by $\xi_{M}(m)=\left.(d / d t)(g(t) \cdot m)\right|_{t=0}$, where $g(t)$ is a curve in $G$ with $g(0)=1$ and $\dot{g}(0)=\xi$; see e.g., [1]. Thus $\rho_{\mathscr{F}(M)}(\xi)=-\xi_{M}$.) Next, let $V$ be a (finite-dimensional) vector space carrying a representation $\rho_{V}$ of $\mathfrak{g}$, let $V^{*}$ be the dual vector space carrying the dual (contragredient) representation $\rho_{V}^{*}$ of $\mathfrak{g}$, and assume we are given a $g$-covariant ( $\rho_{V}^{*}$-equivariant) map

$$
\phi: M \rightarrow V^{*}
$$

from $M$ into $V^{*}$, or equivalently, a $g$-covariant ( $\rho_{V}$-equivariant) linear map

$$
d_{1}: V \rightarrow \mathscr{F}(M)
$$

from $V$ into $\mathscr{F}(M)$; these two maps are related by interchanging their arguments:

$$
d_{1}(v)(m)=\phi(m)(v)
$$

for $m \in M, v \in V$. (One could actually write $d_{1}^{*}$ instead of $\phi$; this notation would be justified by noting that the points in $M$ are precisely the points of $\mathscr{F}(M)$ as a 
commutative algebra, i.e., the continuous homomorphisms of $\mathscr{F}(M)$ into $\mathbb{R}$ : so in that sense, $M$ can be considered as the dual of $\mathscr{F}(M)$ and $d_{1}^{*}$ as the dual of $d_{1}$.) The zero level $C$ of $\phi, C=\phi^{-1}(0)$, can be viewed as a constraint set embedded in $M$, the constraints being the functions $\phi_{i}=d_{1}\left(v_{i}\right)$ with respect to some basis $\left\{v_{i}\right\}_{i}$ of $V$. Due to Eq. (13), $H_{d_{1}}^{0}(K)$ projects onto $\mathscr{F}(C)$, the algebra of functions over $C$ (since $\mathscr{F}(C)=\mathscr{F}(M) / \mathscr{F}_{0}(C), \mathscr{F}_{0}(C)$ being the ideal of functions which vanish on $C)$. Three questions arise naturally in this context, namely as to what are the conditions under which

- $C$ is a submanifold of $M$,

- $H_{d_{1}}^{0}(K)$ is equal to $\mathscr{F}(C)$,

- the Koszul complex $\left(K, d_{1}\right)$ is acyclic.

We are going to show that all three questions have rigorous and very natural answers.

Proposition 1. If $0 \in V^{*}$ is a weakly regular value of $\phi$, then $C$ is (by definition) a submanifold of $M$ and $\mathscr{F}_{0}(C)=\mathscr{F}(M) d_{1}(V)$, implying

$$
H_{d_{1}}^{0}(\mathscr{F}(M) \otimes \Lambda V)=\mathscr{F}(C) .
$$

Sketch of Proof. The proof relies on the observation that the requirement for $0 \in V^{*}$ to be a weakly regular value of $\phi$ is equivalent to the existence of special local coordinates at the points of the submanifold $C$, namely local coordinates of the form $\left(x_{1}, \ldots, x_{p}, x_{p+1}, \ldots, x_{p+q}\right)$, with $p+q=\operatorname{dim} M$ and $q=\operatorname{dim} C$, where the subset $\left(x_{1}, \ldots, x_{p}\right)$ is chosen from the family of constraints $\phi_{i}$ with respect to some basis $\left\{v_{i}\right\}_{i}$ of $V$, while the subset $\left(x_{p+1}, \ldots, x_{p+q}\right)$ forms a system of local coordinates for the submanifold $C$. Moreover, note that $0 \in V^{*}$ is even a regular value of $\phi$ if and only if $p=\operatorname{dim} V^{*}$ (otherwise, $p<\operatorname{dim} V^{*}$ ). The proposition, together with the relation $\mathscr{F}_{01}(C)=\mathscr{F}_{0}(C) \mathscr{F}_{0}(C)\left(\mathscr{F}_{01}(C)\right.$ being the space of functions in $\mathscr{F}_{0}(C)$ which vanish on $C$ together with their first derivatives), now follows from the mean value theorem of real analysis (in its integral form, applied to the first $p$ coordinates, with the last $q$ coordinates as parameters).

Proposition 2 (Poincaré Lemma). Assume that $0 \in V^{*}$ is a weakly regular value of $\phi$. Then it will be even a regular value if and only if the Koszul complex $\left(\mathscr{F}(M) \otimes \Lambda V, d_{1}\right)$ is acyclic.

Proof. Assume first that 0 is a regular value of $\phi$, and observe that the cohomology of the Koszul complex $\left(\mathscr{F}(M) \otimes \Lambda V, d_{1}\right)$ can be computed locally, that is, upon replacing $M$ by (sufficiently small) open subsets $U$ of $M$. (In fact, every $d_{1}$-cocycle and every $d_{1}$-coboundary on $M$ can be suitably localized by means of a partition of unity, because $d_{1}$ vanishes on $\mathscr{P}(M)$.) In the course of the calculation, it is useful to distinguish two cases, according to whether the intersection $U \cap C$ is empty or not. In both cases, however, we prove the vanishing of cohomology by giving a contracting homotopy, i.e. an operator $h: \mathscr{F}(U) \otimes \Lambda^{k} V \rightarrow \mathscr{F}(U) \otimes \Lambda^{k+1} V$ which has the property that the operator $h d_{1}+d_{1} h$ becomes the identity on that part of the complex where the cohomology is supposed to vanish. More precisely, we can construct $h$ in such a way that $\left(h d_{1}+d_{1} h\right) t=t$ for all $t \in \mathscr{F}(U) \otimes \Lambda V$ if 
$U \cap C=\varnothing$ and for all $t \in \mathscr{F}(U) \otimes \Lambda^{+} V$ if $U \cap C \neq \varnothing$, where

$$
\Lambda^{+} V=\bigoplus_{k>0} \Lambda^{k} V .
$$

(The first statement means that the cohomology of $\left(\mathscr{F}(U) \otimes \Lambda V, d_{1}\right)$ vanishes even in degree 0 . when $U \cap C=\varnothing$.) For $U \cap C=\varnothing$, define $h$ by

$$
h=\frac{\sum_{i} \phi_{i} v_{i}}{\sum_{i}\left(\phi_{i}\right)^{2}} .
$$

For $U \cap C \neq \varnothing$, we choose coordinates as indicated above, so that $x_{i}=\phi_{i}$ for $1 \leqq i \leqq p$. Then if $t \in \mathscr{F}(U) \otimes \Lambda^{k} V$ is represented in the form $t=t_{i_{1} \cdots i_{k}} v_{i_{1}} \wedge \cdots \wedge v_{i_{k}}$, define $h t \in \mathscr{F}(U) \otimes \Lambda^{k+1} V$ by

$$
(h t)(x)=\int_{0}^{1} d \lambda \lambda^{k} \partial_{[i} t_{\left.i_{1} \cdots i_{k}\right]}(\lambda \cdot x) v_{i} \wedge v_{i_{1}} \wedge \cdots \wedge v_{i_{k}},
$$

where square brackets on indices are understood to denote full antisymmetrization and $\lambda \cdot x=\left(\lambda x_{1}, \ldots, \lambda x_{p}, x_{p+1}, \ldots, x_{p+q}\right)$. A lengthy but standard calculation shows that $h$ is indeed a contracting homotopy on $\mathscr{F}(U) \otimes \Lambda^{+} V$. For the reverse statement assume 0 to be a weakly regular value of $\phi$ which is not regular, i.e., $p<\operatorname{dim} V^{*}$. Then the differentials $d \phi_{i}$ are linearly dependent on $C$, so there exist functions $f_{i} \in \mathscr{F}(M)$ which do not all vanish on $C$ but satisfy $\left.\sum_{i} f_{i} d \phi_{i}\right|_{C}=0$. This implies $\left.\sum_{i} d\left(f_{i} \phi_{i}\right)\right|_{C}=0$, i.e., $\sum_{i} f_{i} \phi_{i} \in \mathscr{F}_{01}(C)$. Now using $\mathscr{F}_{01}(C)=\mathscr{F}_{0}(C) \mathscr{F}_{0}(C)$, it follows that there exist functions $g_{i}, h_{j} \in \mathscr{F}(M)$ such that

$$
\sum_{i} f_{i} \phi_{i}=\sum_{i, j} g_{i} h_{j} \phi_{j} \phi_{i}
$$

In other words, $\sum_{i}\left(f_{i}-\sum_{j} g_{i} h_{j} \phi_{j}\right) v_{i}$ is a $d_{1}$-cocycle which cannot be a $d_{1}$-coboundary, because $\left.\left(f_{i}-\sum_{j} g_{i} h_{j} \phi_{j}\right)\right|_{C} \neq 0$ for at least one $i$, q.e.d. .

To summarize, we have shown that in the regular case, the BRST cohomology in degree 0 yields precisely the $G$-invariant functions over the constraint manifold $C$, or equivalently, the functions over $C / G$, the space of $G$-orbits in $C$ :

$$
H_{D}^{0}\left(\mathscr{F}(M) \otimes \Lambda V \otimes \Lambda g^{*}\right)=\mathscr{F}(C)^{\mathrm{g}}
$$

(cf. Eqs. (9), (11) and (18)).

\section{Application to Lagrangian Systems}

Historically, BRST cohomology was originally developed as a tool for dealing with degenerate Lagrangian systems whose degeneracy is due to the presence of local symmetries. Here, we want to demonstrate how the construction performed in Sect. 2 can be applied to such systems and; in particular, that it provides a natural framework for incorporating the usual gauge fixing and Faddeev-Popov 
terms appearing, e.g., in Yang-Mills theories (where these terms must be added to the original gauge invariant Lagrangian before Feynman rules can be derived). As mentioned before, we restrict ourselves to systems with finitely many degrees of freedom; moreover, we shall consider only Lagrangians which are strictly gauge invariant, not just quasi-gauge-invariant, i.e., not just gauge invariant up to addition of a total time derivative.

For a Lagrangian system defined over a manifold $Q$ (the configuration space), specified by a Lagrangian $L$ which is a (smooth real) function on the tangent bundle $T Q$ of $Q$, degeneracy means that the fiber derivative $F L$ of $L$ fails to be a local diffeomorphism. More precisely, what is degenerate in such a case is the Hessian, or second fiber derivative, $F^{2} L$ of $L$. (Geometrically, $F L$ is a map $F L: T Q \rightarrow T^{*} Q$, while $F^{2} L$ is a map $F^{2} L: T Q \rightarrow T^{*} Q \vee T^{*} Q$, where $T^{*} Q$ denotes the cotangent bundle of $Q$ and $\vee$ the symmetrized tensor product, so $T^{*} Q \vee T^{*} Q$ is the bundle of covariant symmetric rank 2 tensors over $Q$. Locally, $F L$ is just the usual Legendre transform, where conjugate momenta are defined by taking first derivatives of $L$ with respect to the velocities, while $F^{2} L$ is defined by taking the matrix of second derivatives of $L$ with respect to the velocities:

$$
\begin{gathered}
F L(q, \dot{q})\left(\left.\frac{\partial}{\partial q^{i}}\right|_{q}\right)=\frac{\partial L}{\partial \dot{q}^{i}}(q, \dot{q}), \\
F^{2} L(q, \dot{q})\left(\left.\frac{\partial}{\partial q^{i}}\right|_{q},\left.\frac{\partial}{\partial q^{j}}\right|_{q}\right)=\frac{\partial^{2} L}{\partial \dot{q}^{i} \partial \dot{q}^{j}}(q, \dot{q}) .
\end{gathered}
$$

See [1].) The simplest case - and the only one we shall consider - occurs when the Hessian of $L$ has constant rank on all of $T Q$ : then the zero modes of $F^{2} L$ form an involutive vector subbundle of $T(T Q)$ (in fact, of the vertical bundle $\operatorname{Ver}(T Q)$ ), giving rise to a foliation of $T Q$ whose leaves are the levels $F L^{-1}(q, p)$, $(q, p) \in F L(T Q) \subset T^{*} Q$, of the Legendre transform $F L$.

The standard method to deal with a degenerate Lagrangian system is to regularize it by a choice of gauge. This is normally done by picking a vector-valued function $F: T Q \rightarrow E$ to represent the gauge condition in the form $F=0$. Technically, we shall suppose $E$ to be a Euclidean vector space whose scalar product will be denoted by $\cdot$ and will be used to identify, once and for all, $E$ with its dual $E^{*}$; then we shall call $F$ a gauge function if

- $0 \in E$ is a weakly regular value of $F$,

- the zero level $F^{-1}(0)$ of $F$ intersects every level $F L^{-1}(q, p)$ of $F L$ in at least one point and in at most a discrete set of points.

Under these conditions, it can be shown that the gauge fixed Lagrangian $L_{F}$ on $T Q$ given by

$$
L_{F}=L-\frac{1}{2} F \cdot F
$$

is regular on an open neighbourhood of the zero level $F^{-1}(0)$ of $F$ and that it leads to the same dynamics as the original Lagrangian $L$. Moreover, it is common practice to introduce the vectors in $E$ as auxiliary variables and to pass to an appropriately extended Lagrangian $\hat{L}_{F}$ : these additional (bosonic) degrees of freedom are needed, for example, to define a BRST operator which is nilpotent off-shell. More precisely, one enlarges the configuration space by adding a copy 
of $E$, i.e., by setting $\hat{Q}=Q \times E, T \hat{Q}=T Q \times(E \oplus E)$, and defines $\hat{L}_{F}$ on $T \hat{Q}$ as

$$
\hat{L}_{F}=L+\frac{1}{2} b \cdot b-b \cdot F \text {. }
$$

(Points in $T Q$ are denoted by $(q, \dot{q})$ and points in $T \hat{Q}$ by $(q, \dot{q}, b, \dot{b})$ ).) Obviously, $L_{F}$ can be recovered from $\hat{L}_{F}$ by inserting the equation of motion for $b$ derived from $\hat{L}_{F}$.

Within this general setup, we consider degenerate Lagrangian systems whose degeneracy is entirely due to some kind of gauge invariance. More specifically, we assume that $T Q$ carries an action of a connected Lie group $G$ under which the Lagrangian $L$ is invariant and such that $G$ contains a closed subgroup $G_{\text {loc }}$ whose orbits in $T Q$ are precisely the levels of $F L$. Then letting $G$ act trivially on $E$, we can apply the cohomological method from Sect. 2: take

$$
M=T \hat{Q}=T Q \times(E \oplus E), \quad V=E \oplus E=E^{*} \oplus E^{*}=V^{*},
$$

and let $\phi: M \rightarrow V^{*}$ be the projection onto the second factor. Since $G$ acts trivially on $V\left(\rho_{V}=0\right)$ and is trivially extended from $T Q$ to $M, \phi$ is obviously $g$-covariant; moreover, $\phi$ has $0 \in V^{*}$ as a regular value, with $\phi^{-1}(0)=T Q$. Therefore, Eq. (22) gives

$$
H_{D}^{0}\left(\mathscr{F}(T Q \times(E \oplus E)) \otimes \Lambda(E \oplus E) \otimes \Lambda g^{*}\right)=\mathscr{F}(T Q)^{g} .
$$

(Thus in this version of the BRST complex, the elements of $E$ play a double role: they appear as bosonic degrees of freedom, $(b, \dot{b}) \in M$, and also as fermionic degrees of freedom, $(\eta, \dot{\eta}) \in V$.) In particular, Eq. (28) implies that every $G$-invariant Lagrangian $L$ on $T Q$, being an element of $\mathscr{F}(T Q)^{\mathfrak{g}}$, must correspond to a BRST cohomology class. In general, explicit determination of a BRST cocycle representing this class requires solving the descent equations, but in the present case, this is trivial. Namely, $d_{2} L=0$, so $L$ itself is a solution and every other solution is of the form $\hat{L}^{\mathrm{TOT}}=L+D \Psi$, where $\Psi \in S^{-1}$ is arbitrary. In particular, choosing $\Psi=\eta \cdot\left(\frac{1}{2} b-F\right)$ leads to the well-known form

or

$$
\hat{L}_{F}^{\text {TOT }}=L+\frac{1}{2} b \cdot b-b \cdot F+\eta \cdot D F,
$$

where

$$
\hat{L}_{F}^{\mathrm{TOT}}=L+L_{F}^{\mathrm{GF}}+L_{F}^{\mathrm{FP}},
$$

$$
L_{F}^{\mathrm{GF}}=\frac{1}{2} b \cdot b-b \cdot F
$$

is the gauge fixing term and

$$
L_{F}^{\mathrm{FP}}=\eta \cdot D F
$$

is the Faddeev-Popov term. In this context, the identification of the Lagrangian $L$ with a BRST cohomology class, rather than a BRST cocycle, is the mathematical expression of the requirement that physics should be independent of the choice of gauge.

It should be pointed out that the notion of gauge fixing employed here is rather restrictive, because there is in general no guarantee that a gauge function in the above sense exists. For example, there may be topological obstructions of the type encountered as the "Gribov ambiguity" in Yang-Mills theories. However, the above procedure can be generalized to topologically non-trivial situations if one replaces the vector-valued functions $F$ on $T Q$ by sections of appropriate vector bundles over $T Q$. 


\section{Application to Hamiltonian Systems}

Given the fact that the BRST operator is supposed to characterize observables and physical states, it is natural to consider BRST cohomology not only in the Lagrangian framework but also in the Hamiltonian one. Here, the singular nature of the theory is expressed through the fact that one is dealing with constrained Hamiltonian systems $[6,9,15]$.

To set the stage, recall first that a Hamiltonian system $(P, \omega, H)$ consists of a symplectic manifold $(P, \omega)$ (the phase space) together with a Hamiltonian $H \in \mathscr{F}(P)$. Similarly, a constrained Hamiltonian system $(P, \omega, C, H)$ or $(P, \omega, \phi, H)$ consists of a symplectic manifold $(P, \omega)$ (again called the phase space) together with a submanifold $C$ of $P$ that can be represented as the zero level of a suitable vector-valued function $\phi$ on $P$ (cf. Eq. (1)) and with a Hamiltonian $H \in \mathscr{F}(C)$. In general, the Hamiltonian $H$ is considered as being extended to the entire phase space $P$, but such an extension is of course far from unique. In fact, if (as usual) 0 is supposed to be a weakly regular value of $\phi$, then Proposition 1 shows that any two such extensions will differ by a linear combination of the constraints (with coefficients in $\mathscr{F}(P))$; they should be physically indistinguishable. The BRST procedure, however, cannot be directly applied to arbitrary constrained Hamiltonian systems but only to those which are, roughly speaking, "first class." This means that a) the constraints close under Poisson brackets, viz.

$$
\left\{\phi_{i}, \phi_{j}\right\}=f_{i j}^{k} \phi_{k},
$$

with structure functions $f_{i j}^{k} \in \mathscr{F}(P)$, and b) the Hamiltonian reproduces the constraints under Poisson brackets, viz.

$$
\left\{H, \phi_{i}\right\}=h_{i}^{k} \phi_{k},
$$

with structure functions $h_{i}^{k} \in \mathscr{F}(P)$. In more invariant algebraic terms, Eqs. (33) and (34) can be restated as saying that the ideal $\mathscr{F}_{0}(C)$ in $\mathscr{F}(P)$ consisting of those functions on $P$ that vanish on $C$ has the property that a)

$$
\left\{\mathscr{F}_{0}(C), \mathscr{F}_{0}(C)\right\} \subset \mathscr{F}_{0}(C),
$$

i.e., $\mathscr{F}_{0}(C)$ is a Poisson subalgebra of $\mathscr{F}(P)$, and b)

$$
\left\{H, \mathscr{F}_{0}(C)\right\} \subset \mathscr{F}_{0}(C),
$$

i.e., $H$ normalizes $\mathscr{F}_{0}(C)$ under Poisson brackets. (Note that the first condition implies that the second condition does not depend on the choice of extension of $H$ off $C$.) Geometrically, Eqs. (33) and (35) mean that $C$ is a coisotropic submanifold of $P$, while Eqs. (34) and (36) say that $H$ can be chosen so that on $C$, the corresponding Hamiltonian vector field $X_{H}$ is tangent to $C$.

As in the Lagrangian case, a particular role is played by constrained Hamiltonian systems in which the constraints are the result of "local" symmetries. The appropriate concept for this kind of situation is that of a Hamiltonian $G$-space $(P, \omega, G, J)$, which consists of a symplectic manifold $(P, \omega)$ together with a connected Lie group $G$ with Lie algebra $g$ such that $G$ acts on $P$ by symplectic diffeomorphisms and

$$
J: P \rightarrow \mathrm{g}^{*}
$$


is a $G$-covariant ( $\mathrm{Ad}^{*}$-equivariant) momentum mapping for this action [1]. In this case, the natural choice for defining a constrained Hamiltonian $G$-space is to take $\phi=J$ so that the constraint manifold is the zero level $C=J^{-1}(0)$ of the momentum mapping. (Indeed, considering $G$ as a local symmetry and not just a global one forces the trajectories of the system to be constrained to the zero level of the momentum mapping: this has first been observed in the context of Yang-Mills theories and of general relativity but is in fact quite a general feature; see, e.g., $[2,7]$ and references therein.) What remains to be added to fix the dynamics of the theory is the choice of an invariant Hamiltonian $H$. When there are no constraints, $H$ is simply supposed to be a $G$-invariant function on $P$, while in the case where the system is constrained to the zero level $J^{-1}(0)$ of the momentum mapping, $H$ should be a $G$-invariant function on $J^{-1}(0)$. This function can, as mentioned above, be extended to a function on the entire phase space $P$, but the extension need not be $G$-invariant. (If $G$ is compact, then one can always find a $G$-invariant extension.) Also, with this choice $G$-covariance of the momentum mapping $J$ and $G$-invariance of the Hamiltonian $H$ force Eqs. (33) and (34) to be satisfied, the $f_{i j}^{k}$ here being the structure constants of $\mathfrak{g}$. The observables of the system are not the functions on $J^{-1}(0)$ but rather on the reduced phase space $P_{0}$ obtained from $J^{-1}(0)$ by taking the quotient with respect to the action of $G$ :

$$
P_{0}=J^{-1}(0) / G
$$

Now the interpretation of BRST cohomology given in [13] is quite natural: take $M=P, V=\mathfrak{g}$ and $\phi=J$. Then according to the results obtained in Sect. 2, we have the equality

$$
H_{D}^{0}\left(\mathscr{F}(P) \otimes \Lambda \mathfrak{g} \otimes \Lambda \mathfrak{g}^{*}\right)=\mathscr{F}\left(J^{-1}(0)\right)^{\mathfrak{g}}=\mathscr{F}\left(P_{0}\right),
$$

and hence the BRST cohomology in degree 0 gives precisely the observables of the system, if and only if $0 \in \mathrm{g}^{*}$ is a regular value of $J$.

Proposition 3. $0 \in \mathfrak{g}^{*}$ is a regular value of $J$ if and only if $G$ acts almost freely on $J^{-1}(0)$.

Proof. $G$ acts almost freely on $J^{-1}(0)$ if and only if for any $X \in \mathfrak{g}$, the fundamental vector field $X_{P}$ on $P$ corresponding to $X$ does not vanish anywhere on $J^{-1}(0)$, that is, if for any point $p \in J^{-1}(0)$ and any $X \in \mathfrak{g}, d J(X)(p) \neq 0$, which means exactly that at any point $p \in J^{-1}(0), T_{p} J$ is onto, q.e.d..

This shows the requirement that $0 \in \mathfrak{g}^{*}$ should be a regular value of $J$ to be very restrictive: it is therefore a natural and important problem to extend the BRST cohomological constructions to cover more general situations, such as the one where $G$ acts on $J^{-1}(0)$ in such a way that all isotropy groups are conjugate.

As in the Lagrangian case, Eq. (39) implies that every $G$-invariant Hamiltonian $H$ on $J^{-1}(0)$, being an element of $\mathscr{F}\left(J^{-1}(0)\right)^{9}$, must correspond to a BRST cohomology class. Again, explicit determination of a BRST cocycle representing this class requires solving the descent equations. When $H$ is extended to $P$ in a $G$-invariant manner, this is trivial, since then $d_{2} H=0$, so $H$ itself is a solution. Otherwise, the solution may be complicated, but up to BRST coboundaries, it will lead to the same result as the formalism of Batalin, Fradkin and Vilkovisky [3]. 


\section{Realization of Local Symmetries Through Finite-Dimensional Lie Groups}

In the standard approach to Lagrangian systems with local symmetries, the symmetry group is viewed as an infinite-dimensional group $\mathscr{G}$ acting on the infinite-dimensional space $\mathscr{2}$ of all possible trajectories. For typical systems with finitely many degrees of freedom and configuration space $Q$, the space $\mathscr{Q}$ is more or less fixed, while for the group $\mathscr{G}$, one encounters two essentially distinct possibilities. Namely, if $I \subset \mathbb{R}$ denotes some parameter interval fixed one and for all (e.g., $I=[0,1]$ or $I=\mathbb{R}), \mathscr{Q}$ is the space $C^{\infty}(I, Q)$ of (smooth) paths in $Q$ or a subspace thereof (usually defined by imposing suitable boundary conditions), while $\mathscr{G}$ is

- either a subgroup of the group $\operatorname{Diff}_{0}(I)$ of (smooth) orientation preserving diffeomorphisms of $I$,

- or a subgroup of the group $C^{\infty}(I, G)$ of (smooth) paths in some finite-dimensional connected Lie group $G$ acting on $Q$.

In both cases, we want to argue that invariance of the action

$$
S[q]=\int d t L(q(t), \dot{q}(t))
$$

under $\mathscr{G}$ can be reformulated as covariance or even invariance of the Lagrangian $L$ under an appropriate finite-dimensional connected Lie group $\widetilde{G}$ acting on $T Q$.

The first case, where $\mathscr{G} \subset \operatorname{Diff}_{0}(I)$ acts by transformation of the independent variable (the argument) according to

$$
(\sigma \cdot q)(t)=q\left(\sigma^{-1}(t)\right)
$$

for $\sigma \in \operatorname{Diff}_{0}(I), q \in C^{\infty}(I, Q), t \in I$, covers systems defined by an action which is reparametrization invariant, the standard example being the relativistic point particle. It is well known [14] that these are exactly the actions derived from Lagrangians which are homogeneous of degree 1 in the velocities, or equivalently, which are covariant under the one-dimensional Weyl group that acts trivially on the positions and just rescales the velocities. Thus in the present context, the Weyl group is a copy of the additive group $\mathbb{R}$ which acts on $T Q$ according to

$$
\alpha \cdot(q, \dot{q})=\left(q, e^{\alpha} \dot{q}\right),
$$

for $\alpha \in \mathbb{R},(q, \dot{q}) \in T Q$, and covariance of $L$ under the Weyl group means that

$$
L(\alpha \cdot(q, \dot{q}))=e^{\alpha} L(q, \dot{q}),
$$

for $\alpha \in \mathbb{R},(q, \dot{q}) \in T Q$. Unfortunately, the BRST cohomological constructions discussed in Sects. 2 and 3 are tailored to invariant Lagrangians and are therefore not directly applicable to the present situation; they should be extended to cover this more general case as well.

The second case, where $\mathscr{G} \subset C^{\infty}(I, G)$ acts by transformation of the dependent variable according to

$$
(g \cdot q)(t)=g(t) \cdot q(t)
$$

for $g \in C^{\infty}(I, G), q \in C^{\infty}(I, Q), t \in I$, is the one of interest to us. Here, the group $\tilde{G}$ 
with its action on $T Q$ can be constructed explicitly from the group $G$ with its action on $Q$; it is the Lie algebra of this group $\widetilde{G}$, rather than that of the original group $G$ itself, which has to be inserted into the BRST complex considered in Sect. 3. In the following, we are not going to give a completely general treatment, but shall discuss two classes of models which can both be viewed as finite-dimensional (mechanical) analogues of gauge theories of the Yang-Mills type: the first class corresponds to the Yang-Mills sector, while the second class mimics the matter sector. This implies that in the first case, gauge invariance is manifest from the beginning, while in the second case, we start out from a global symmetry and obtain gauge invariance as a result of the procedure of "gauging the global symmetry": thus we expect that we must enlarge the configuration space by introducing a Lagrange multiplier $\lambda$ which is nothing but the (mechanical) analogue of the Lie-algebra-valued gauge potential.

Common to both classes of models to be discussed is that we begin with a manifold $Q$ as the original configuration space and with an action of a finite-dimensional connected Lie group $G$ on $Q$. This action of $G$ on $Q$ can be lifted to an action of $G$ on $T Q$ by putting, for any curve $q(\cdot)$ in $Q$,

$$
\left.g \cdot \frac{d}{d t} q(t)\right|_{t=0}=\left.\frac{d}{d t}(g \cdot q(t))\right|_{t=0},
$$

together with

$$
g \cdot(q, \dot{q})=(g \cdot q, g \cdot \dot{q}) .
$$

In what follows, a Lagrangian $L$ on $T Q$ will be called globally G-invariant if it is invariant under this lifted action. In infinitesimal form, this condition can be written as $X_{T Q} L=0$ for all $X \in \mathfrak{g}$, where $X_{T Q}$ denotes the fundamental vector field on $T Q$ corresponding to $X \in \mathfrak{g}$.

The first class of models is obtained by applying the tangent functor once, i.e., by making use of the fact that the lifted action of $G$ on $T Q$ extends naturally to an action of the tangent group $T G$ on $T Q$. Indeed, the tangent map $T G \times T G \rightarrow T G$ to the group multiplication in $G$ (viewed as a map $G \times G \rightarrow G$ ) defines a group multiplication in $T G$. Similarly, the tangent map $T G \times T Q \rightarrow T Q$ to the group action of $G$ on $Q$ (viewed as a map $G \times Q \rightarrow Q$ ) defines a group action of $T G$ on $T Q$. Now observe that as a vector bundle over $G$, the tangent bundle $T G$ of $G$ is globally trivial: one can use either left translations or right translations to write down an explicit trivialization $T G \cong G \times \mathfrak{g}$. We shall employ right translations and thus identify $T G$ with $G \times \mathfrak{g}$ by identifying $(g, \dot{g})=(g, X g) \in T G$ with $(g, X) \in G \times \mathfrak{g}$. Then as a group, $T G \cong G \triangleright \mathfrak{g}$, where $\triangleright$ denotes taking the semidirect product; explicitly, the group multiplication in $G \triangleright \mathfrak{g}$ and the group action of $G \triangleright \mathfrak{g}$ on $T Q$ are given by

$$
\left(g_{1}, X_{1}\right)\left(g_{2}, X_{2}\right)=\left(g_{1} g_{2}, X_{1}+\operatorname{Ad}\left(g_{1}\right) X_{2}\right),
$$

and

$$
(g, X) \cdot(q, \dot{q})=\left(g \cdot q, g \cdot \dot{q}+X_{Q}(g \cdot q)\right),
$$

respectively, where $X_{Q}$ denotes the fundamental vector field on $Q$ corresponding to $X \in \mathfrak{g}$. 
Proposition 4. A Lagrangian $L$ on $T Q$ is invariant under the action of $\widetilde{G}=T G$ if and only if for every path $q(\cdot) \in C^{\infty}(I, Q)$ in $Q$, the function $L(q(\cdot), \dot{q}(\cdot)) \in C^{\infty}(I)$ is invariant under the action of $C^{\infty}(I, G)$. Therefore, we shall call such a Lagrangian $L$ locally G-invariant.

Proof. Let $g \in C^{\infty}(I, G), q \in C^{\infty}(I, Q)$ and $X \in C^{\infty}(I, \mathfrak{g})$, where $X(s)=\left.\frac{d}{d t}\left(g(t) g^{-1}(s)\right)\right|_{t=s}$. Then

$$
\begin{aligned}
\left.\frac{d}{d t}(g \cdot q)(t)\right|_{t=s} & =\left.\frac{d}{d t}(g(t) \cdot q(s))\right|_{t=s}+\left.\frac{d}{d t}(g(s) \cdot q(t))\right|_{t=s} \\
& =\left.\frac{d}{d t}\left(g(t) g^{-1}(s) g(s) \cdot q(s)\right)\right|_{t=s}+\left.g(s) \cdot \frac{d}{d t} q(t)\right|_{t=s} \\
& =X(s)_{Q}(g(s) \cdot q(s))+\left.g(s) \cdot \frac{d}{d t} q(t)\right|_{t=s},
\end{aligned}
$$

that is

$$
\left((g \cdot q)(s),\left.\frac{d}{d t}(g \cdot q)(t)\right|_{t=s}\right)=(g(s), X(s)) \cdot\left(q(s),\left.\frac{d}{d t} q(t)\right|_{t=s}\right) .
$$

The proposition follows directly from this formula, q.e.d..

In the case of a regular action of $G$ on $Q$, where the orbit space $Q / G$ is a manifold [1], a nice interpretation of the above situation can be given. Namely the $T G$-invariant Lagrangians on $T Q$ are in one-to-one correspondence with the Lagrangians on $T(Q / G)$. Thus the physical configuration space is in fact $Q / G$ and the action of $G$ cannot be observed.

The second class of models provides a natural answer to the question how to construct locally $G$-invariant Lagrangians from globally $G$-invariant ones. To this end, we construct an extended configuration space, together with an extended symmetry group, enlarging both $Q$ and $G$ by a copy of $\mathrm{g}$ :

$$
\hat{Q}=Q \times \mathfrak{g}, \quad \hat{G}=G \triangleright \mathfrak{g} .
$$

By definition, the group multiplication in $\widehat{G}$ is (as indicated by the notation) that for the semidirect product (cf. Eq. (47)) and the group action of $\hat{G}$ on $\hat{Q}$ is given by

$$
(g, Z) \cdot(q, \lambda)=(g \cdot q, Z+\operatorname{Ad}(g) \lambda) .
$$

Applying the tangent functor to $\hat{G}$ and $\hat{Q}$ as above leads to an action of $T \hat{G}$ on $T \hat{Q}$. Note that $T \hat{G}$ can be viewed as the double tangent group $T(T G)=T^{2} G$ of $G$ but can also be identified with the semidirect product $(G \triangleright \mathfrak{g}) \triangleright(\mathfrak{g} \triangleright \mathfrak{g})$, while $T \hat{Q}$ can be identified with $T Q \times(\mathfrak{g} \oplus \mathrm{g})$. Combining Eqs. (47), (48) and (50), we infer that the group multiplication in $(G \triangleright \mathfrak{g}) \triangleright(\mathfrak{g} \triangleright \mathfrak{g})$ and the group action of $(G \triangleright \mathfrak{g}) \triangleright(\mathfrak{g} \triangleright \mathfrak{g})$ on $T Q \times(\mathfrak{g} \oplus \mathfrak{g})$ are given by

$$
\begin{aligned}
& \left(g_{1}, Z_{1}, X_{1}, Y_{1}\right)\left(g_{2}, Z_{2}, X_{2}, Y_{2}\right) \\
& \quad=\left(g_{1} g_{2}, Z_{1}+\operatorname{Ad}\left(g_{1}\right) Z_{2}, X_{1}+\operatorname{Ad}\left(g_{1}\right) X_{2}, Y_{1}+\operatorname{Ad}\left(g_{1}\right) Y_{2}+\left[Z_{1}, \operatorname{Ad}\left(g_{1}\right) X_{2}\right]\right),
\end{aligned}
$$


and

$$
\begin{aligned}
& (g, Z, X, Y) \cdot(q, \lambda, \dot{q}, \dot{\lambda}) \\
& \quad=\left(g \cdot q, Z+\operatorname{Ad}(g) \lambda, g \cdot \dot{q}+X_{Q}(g \cdot q), \operatorname{Ad}(g) \dot{\lambda}+Y+[X, Z+\operatorname{Ad}(g) \lambda]\right),
\end{aligned}
$$

respectively. But this is not exactly what we want, since it describes a locally $\hat{G}$-invariant system over $T \hat{Q}$ instead of a $G$-invariant one over $T Q$. It turns out that the right symmetry group to look at is not $T^{2} G$ but the subgroup $T^{[2]} G$ consisting of those elements that define a second order differential equation on $G$ [1]. Under the identification

we get

$$
T^{2} G \cong(G \triangleright \mathfrak{g}) \triangleright(\mathfrak{g} \triangleright \mathfrak{g})
$$

$$
T^{[2]} G \cong\{(g, Z, X, Y) \in(G \triangleright \mathfrak{g}) \triangleright(\mathfrak{g} \triangleright \mathfrak{g}) \mid Z=X\} .
$$

Proposition 5. A Lagrangian $\hat{L}$ on $T \hat{Q}$ is invariant under the action of $G=T^{[2]} G$ if and only if for every path $(q(\cdot), \lambda(\cdot)) \in C^{\infty}(I, \hat{Q})$ in $\hat{Q}$, the function $\hat{L}(q(\cdot), \lambda(\cdot), \dot{q}(\cdot)$, $\lambda(\cdot)) \in C^{\infty}(I)$ is invariant under the action of $C^{\infty}(I, G) \subset C^{\infty}(I, \hat{G})$. Again, we shall call such a Lagrangian $\hat{L}$ locally $G$-invariant.

Proposition 6. Every globally G-invariant Lagrangian $L$ on $T Q$ defines a locally will therefore be omitted.

The construction is completed by observing that one can identify globally $G$-invariant Lagrangians on $T Q$ with locally $G$-invariant ones on $T \hat{Q}$.

Proposition 6. Every globally G-invariant Lagrangian $L$ on $T Q$ defines a locally G-invariant Lagrangian $\hat{L}$ on $T \hat{Q}$ according to

$$
\hat{L}(q, \lambda, \dot{q}, \dot{\lambda})=L\left(q, \dot{q}-\lambda_{Q}(q)\right) .
$$

Conversely, every locally G-invariant Lagrangian $\hat{L}$ on $T \hat{Q}$ arises in this way from $a$ globally G-invariant Lagrangian L on TQ: just put

$$
L(q, \dot{q})=\hat{L}(q, 0, \dot{q}, 0) .
$$

Proof. Let $L$ be a globally $G$-invariant Lagrangian on $T Q$, and let $\hat{L}$ be defined by Eq. (55); then

$$
\begin{aligned}
\widehat{L} & ((g, X, X, Y) \cdot(q, \lambda, \dot{q}, \dot{\lambda})) \\
& =\hat{L}\left(g \cdot q, X+\operatorname{Ad}(g) \lambda, g \cdot \dot{q}+X_{Q}(g \cdot q), \operatorname{Ad}(g) \dot{\lambda}+Y+[X, \operatorname{Ad}(g) \lambda]\right) \\
& =L\left(g \cdot q, g \cdot \dot{q}+X_{Q}(g \cdot q)-(X+\operatorname{Ad}(g) \lambda)_{Q}(g \cdot q)\right) \\
& =L\left(q, \dot{q}-g^{-1} \cdot(\operatorname{Ad}(g) \lambda)_{Q}(g \cdot q)\right) \\
& =L\left(q, \dot{q}-\lambda_{Q}(q)\right) \\
& =\hat{L}(q, \lambda, \dot{q}, \dot{\lambda}),
\end{aligned}
$$

so $\hat{L}$ is locally $G$-invariant. (In the third step we have used that $L$ is globally $G$-invariant.) Conversely, let $\hat{L}$ be a locally $G$-invariant Lagrangian on $T \hat{Q}$; then

$$
\begin{aligned}
\hat{L}(q, \lambda, \dot{q}, \dot{\lambda}) & =\hat{L}\left((1, \lambda, \lambda, \dot{\lambda}) \cdot\left(q, 0, \dot{q}-\lambda_{Q}(q), 0\right)\right) \\
& =\hat{L}\left(q, 0, \dot{q}-\lambda_{Q}(q), 0\right),
\end{aligned}
$$

thus proving the validity of Eq. (56). 
Clearly, the variable $\lambda$ acts as a Lagrange multiplier, because $\hat{L}$ does not depend on the corresponding velocity $\lambda$.

Acknowledgement. The authors wish to thank all members of the Freiburg theory group, and in particular C. Emmrich, for helpful discussions.

\section{References}

1. Abraham, R., Marsden, J. E.: Foundations of mechanics, second edition, updated 1985 printing. Menlo Park: Benjamin Cummings 1978

2. Arms, J. M., Marsden, J. E., Moncrief, V.: Symmetry and bifurcations of momentum mappings. Commun. Math. Phys. 78, 455-478 (1981)

3. Fradkin, E. S., Vilkovisky, G.: Quantization of relativistic systems with constraints. Phys. Lett. 55B, 224-226 (1975); Batalin, I. A., Vilkovisky, G.: Relativistic $S$-matrix of dynamical systems with Boson and Fermion constraints. Phys. Lett. 69B, 309-312 (1977)

4. Becchi, C., Rouet, A., Stora, R.: The Abelian Higgs Kibble Model, Unitarity of the S-Operator. Phys. Lett. 52B, 344-354 (1974); Tyutin, I. V.: Report Fian 39, (1975) (unpublished)

5. Bott, R., Tu, L. W.: Differential forms in algebraic topology. Berlin, Heidelberg, New York: Springer 1982

6. Dirac, P. A. M.: Lectures on quantum mechanics. Belfer Graduate School of Science Monograph Series, No. 2, New York, 1964

7. Emmrich, C., Römer, H.: Orbifolds as configuration spaces of systems with gauge symmetries. Commun. Math. Phys. 129, 69-94 (1990)

8. Faddeev, L. D., Popov, V. N.: Feynman diagrams for the Yang-Mills field. Phys. Lett. 25B, 29-30 (1967)

9. Hanson, A., Regge, T., Teitelboim, C.: Constrained Hamiltonian systems. Roma: Academia Nazionale dei Lincei, 1976

10. Henneaux, M.: Hamiltonian form of the path integral for theories with a gauge freedom. Phys. Rep. 126, No.1, 1-66 (1985)

11. Henneaux, M., Teitelboim, C.: BRST cohomology in classical mechanics. Commun. Math. Phys. 115, 213-230 (1988)

12. Fish, J., Henneaux, M., Stasheff, J., Teitelboim, C.: Existence, uniqueness and cohomology of the classical BRST charge with ghosts of ghosts. Commun. Math. Phys. 120, 379-407 (1989)

13. Kostant, B., Sternberg, S.: Symplectic reduction, BRS cohomology, and infinite-dimensional Clifford algebras. Ann. Phys. 176, 49-113 (1987)

14. Rund, H.: The Hamilton-Jacobi theory in the calculus of variations. Van Nostrand, 1966

15. Sundermeyer, K.: Constrained dynamics. Lecture Notes in Physics vol. 169, Berlin, Heidelberg, New York: Springer 1982

16. Tate, J.: Homology of Noetherian rings and local rings. Illinois J. Math. 1, 14-27 (1957)

Communicated by N. Y. Reshetikhin 
